\title{
Resistencia a la compresión del concreto con adición del poliestireno expandido y extruido reciclados
}

\author{
Compressive strength of concrete with the addition of recycled expanded and \\ extruded polystyrene
}

\author{
Janet Andía Arias ${ }^{1}$, Raymundo Erazo Erazo² \\ Recibido: 28/01/2021 - Aprobado: 25/10/2021 - Publicado: 23/12/2021
}

\begin{abstract}
RESUMEN
El objetivo de la presente investigación fue evaluar la resistencia a la compresión del concreto de $350 \mathrm{~kg} / \mathrm{cm}^{2}$ de diseño fabricado en la ciudad de Huancayo, utilizando agregados gruesos y finos con materiales reciclados de poliestirenos extruido (XPS) lavado y sin lavar debidamente rayado y poliestireno expandido (EPS) sin lavar en forma de perlas. Se tomaron muestras de residuos de poliestireno en instalaciones recreacionales y de servicios públicos y los agregados de canteras próximas a la ciudad de Huancayo que fueron sometidos a ensayos de control de calidad según la norma técnica peruana NTP 400.037. Se realizaron diferentes mezclas constituidas por poliestirenos reciclados y los agregados para la fabricación de concreto, la resistencia a la compresión se analizó en un laboratorio especializado. Los resultados demostraron que la mezcla de concreto fabricado con XPS reciclado y lavado, cuya proporción fue $30 \%$ de agregado grueso por XPS y $20 \%$ de agregado fino por XPS, a la edad de 28 días, superó la resistencia de diseño de $350 \mathrm{~kg} / \mathrm{cm}^{2}$, obteniéndose un valor de $365 \mathrm{~kg} / \mathrm{cm}^{2}$ siendo aproximadamente un $74 \%$ respecto de la resistencia a la compresión de la muestra patrón de mezcla sin materiales reciclados, cuyo valor es $495 \mathrm{~kg} / \mathrm{cm}^{2}$.
\end{abstract}

Palabras claves: Poliestireno extruido reciclado; poliestireno expandido reciclado; contaminación ambiental; concreto fabricado con residuos sólidos.

\begin{abstract}
The objective of this research was to evaluate the compressive strength of the $350 \mathrm{~kg} / \mathrm{cm}^{2}$ design concrete manufactured in the city of Huancayo, using coarse and fine aggregates with recycled extruded polystyrene (XPS) materials, washed and unwashed, duly scratched unwashed expanded polystyrene (EPS) in pearl shape. Samples of polystyrene waste were taken in recreational and public service facilities and aggregates from quarries near the city of Huancayo that were subjected to quality control tests according to the Peruvian technical standard NTP 400.037. Different mixtures consisting of recycled polystyrenes and aggregates were made for the manufacture of concrete, the compressive strength was analyzed in a specialized laboratory. The results showed that the concrete mix made with recycled and washed XPS, whose proportion was $30 \%$ coarse aggregate

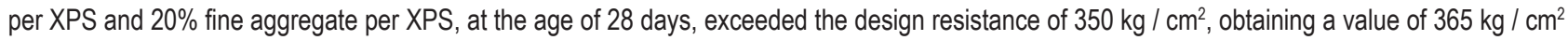
being approximately $74 \%$ with respect to the resistance to compression of the standard mixture sample without recycled materials, whose value is $495 \mathrm{~kg} / \mathrm{cm}^{2}$.
\end{abstract}

Keywords: Recycled extruded polystyrene; recycled expanded polystyrene; environmental pollution; concrete made with solid waste.

\footnotetext{
1 Universidad Nacional Mayor de San Marcos, . Facultad de Ingeniería Geológica, Minera, Metalúrgica y Geográfica, Unidad de Posgrado, Lima, Perú. E-mail: janetsitaand@gmail.com - ORCID: https://orcid.org/0000-0002-6084-0672

2 Universidad Nacional Mayor de San Marcos, Facultad de Química e Ingeniería Química, Departamento Académico de Procesos. E-mail: rerazoe@unmsm.edu.pe - ORCID: https://orcid.org/0000-0003-1480-7641
} 


\section{INTRODUCCIÓN}

En la ciudad de Huancayo, los bienes de consumo y servicio fabricados con poliestireno expandido y poliestireno extruido, luego de su uso se constituyen en residuos sólidos, que actualmente, no se reciclan y prácticamente el $100 \%$ terminan en los botaderos, resultando de este modo en un grave problema ambiental, la densidad del poliestireno es muy baja, entre 10 a $50 \mathrm{~kg} / \mathrm{cm}^{3}$, por lo que la contaminación visual generada es alta. La definición de residuo sólido es una noción activa que desarrolla paralelamente el progreso económico y productivo (OEFA, 2014); (Montes C. 2009). El reciclado de materiales desde cualquier punto de vista es una idea atractiva y necesaria. En lo ambiental, no solo tolera dar nuevo uso a esos "residuos" que en un inicio ya habrían dedicado la función para el cual llevaron manufacturados, sino también reducir la magnitud de la colocación en los rellenos sanitarios urbanos y sub-urbanos (Álvarez, 2010); (Villafuerte et al., 2014).

Los restos poliméricos, incorporado el poliestireno expandido, EPS, contribuyen 2.000 millones de toneladas de $\mathrm{CO} 2$, semejante al año en transmisión de gases de efecto invernadero a la atmósfera. (Rodríguez et al., 2014). En cuanto a EE. UU y Europa el reutilizar estos residuos sólidos se ha considerado en dos elecciones principales: la primera es la valorización como energía, por el cual se incineran los residuos aprovechando el calor liberado para su transformación en energía mecánica y finalmente en energía eléctrica; y la segunda alternativa es el reciclado mecánico que consiste en la trituración y compactación, (Betancourt Sánchez, 2015). Sin embargo, algunos argumentos ambientales, como la generación de emisiones tóxicas, se están constituyendo como una oposición pública oponiéndose al proceso de cremación. Por otra parte, el reciclado mecánico, a pequeño resulta más costoso que los polímeros vírgenes. Es necesario investigar otras alternativas de ejecución para minimizar los costos de los 2 procesos de reciclaje (Rojas, 2014); (Betancourt D, \& Solano M, 2016).

El impacto ambiental se conceptúa como las implicancias que sobre la sociedad y la economía poseen las categorías ambientales. (Villafuerte et al., 2014).

La Fundación Verde Natura con el lema "reciclamos EPS para un mundo mejor" (Natura, 2019), es una de las fundaciones colombianas sin fines de lucro, que aporta a la sociedad una elección de reciclaje del poliestireno extruido (XPS) y poliestireno expandido (EPS), por sus siglas en inglés, que ayudan a reducir el daño al medio ambiente, menorando estos residuos sólidos. Para ello, con los actores comprometidos directamente, han detallado líneas de reciclaje para el desarrollo del plástico desde su producción hasta su disposición final; allí acogen y acumular los residuos para manejarlos y por último recuperar una resina de particularidad semejante a los plásticos inéditos, incrementando el periodo de vida de los materiales (Natura, 2019).

Por otro lado, el poliestireno tiene una degradación muy lenta a las condiciones ambientales.
(McCauley, 2015) , profesor de biología marina en la Universidad de California, Santa Bárbara, EE. UU., estudió las causas mecánicas y químicas del daño a las especies marinos por el poliestireno expandido - EPS. La degradación ambiental de poliestireno (por ejemplo, por bacterias) conduce a la producción de fragmentos pequeños denominados microplásticos, que permanecen durante mucho tiempo en el medio ambiente (por ejemplo, en sistemas marinos) y causan efectos adversos en la salud de los peces, pues confunden las partículas de poliestireno con fuentes de alimentación.

En el botadero de Huancayo se han observado que las garzas y otros animales consumen el poliestireno extruido y expandido, aún no hay estudios del daño que causan a su organismo.

El propósito de esta investigación es evaluar la dureza a la comprensión del concreto fabricado en la ciudad de Huancayo utilizando porcentajes del poliestireno expandido y poliestireno extruido reciclados desde los residuos sólidos urbanos. El procedimiento consistió en realizar ensayos de laboratorio para hallar las propiedades mecánicas de los agregados que intervinieron para el concreto, a fin de ver el cumplimiento de los requisitos que deben cumplir los agregado según la norma técnica peruana NTP 400.037, para lo cual eso realizó el reemplazo por un $30 \%$ en agregado grueso y un $20 \%$ en agregado fino con el poliestireno extruido (lavado y sin lavar, ambos rayados con un rayador doméstico) y el poliestireno expandido (en forma de perlas limpio) reciclados a fin de obtener concretos de resistencias mayores a $350 \mathrm{~kg} / \mathrm{cm}^{2}$.

El estudio realizado va a contribuir en la gestión ambiental del aprovechamiento de los residuos sólidos industriales y domésticos, mediante procesos de reciclado, para lo cual se deben promover mecanismos de aprovechamiento apropiados.

\section{MÉTODOS}

Las muestras del poliestireno extruido y poliestireno expandido fueron obtenidas por un muestreo no aleatorio de residuos sólidos en la ciudad de Huancayo, tanto en las instalaciones de recreación social, actividades culturales, comercios, restaurantes, entre otros (Parres García, 2005), obteniéndose un promedio de $1 \mathrm{~kg}$ del poliestireno extruido y $1 \mathrm{~kg}$ del poliestireno expandido. Luego, la mitad del poliestireno extruido se procedió a lavar con detergente de uso doméstico y puesto a secado para luego reducirlo de tamaño por rayado para su almacenamiento y posterior uso en la fabricación de concreto; la otra mitad, sin lavar, se sometió a limpieza con un material absorbente, para su posterior rayado. En el caso del poliestireno expandido, solo se procedió a una limpieza superficial, para luego ser desintegrarlo en forma de perlas para su posterior uso.

El agregado grueso procede de la cantera del Río Mantaro - Anexo Santa Rosa - Distrito de Huamancaca Chico y el agregado fino del Río Mantaro Puente Las Palmas, Distrito de Orcotuna, obteniéndose $400 \mathrm{~kg}$ de agregado grueso y $400 \mathrm{~kg}$ de agregado fino, los cuales se llevaron al laboratorio de ensayos físico-químicos y mecánicos para 
los análisis de calidad de los agregados de acuerdo con la norma NTP 400.037: ensayos de granulometría, impurezas orgánicas, alcalinidad, sulfatos, durabilidad al sulfato de magnesio, cloruros, carbonatación, abrasión de los Ángeles y equivalente de arena. Con estos datos se procedió a realizar el diseño de mezcla por resistencia por el método de finura de $350 \mathrm{~kg} / \mathrm{cm}^{2}$, concretos utilizados en columnas, vigas, losas aligeradas, losas macizas, zapatas, losas para puentes, etc.

Para la preparación de las muestras de concreto, se diseñaron probetas cilíndricas de 4" x 8", en donde se realizó la combinación de reemplazo del agregado fino 20 $\%$ y agregado grueso $30 \%$, por poliestireno extruido lavado y sin lavar y por poliestireno expandido respectivamente. La resistencia a la compresión se llevó a cabo en distintas edades de tres, siete, catorce, veintiuno y veintiocho días. La resistencia a la compresión de probetas se ejecutó según la norma ASTM C39/C39M -12, empleándose una prensa de concreto de una calibración no más de 1 año.

Luego de realizado el diseño de mezcla teórico, se ejecutó el diseño de mezcla práctico del concreto patrón el agregado grueso y fino, agua y cemento sin reemplazo del poliestireno extruido o del poliestireno expandido. Se preparó 3 probetas por cada edad de 3, 7, 14, 21 y 28 días procediéndose al curado de las probetas de concreto para su posterior ensayo de resistencia a la compresión de muestras cilíndricas de concreto.

Del mismo modo, se procedió a realizar el diseño de mezcla práctico con los siguientes porcentajes de reemplazo por volumen del $20 \%$ del agregado fino y $30 \%$ del agregado grueso; $30 \%$ del agregado grueso y $40 \%$ del agregado fino; $60 \%$ del agregado fino y $70 \%$ del agregado fino (todos los reemplazo fueron porcentaje en volumen): Para las combinaciones con poliestireno extruido lavado, con poliestireno extruido sin lavar, con poliestireno expandido, se preparó 2 probetas de concreto por cada edad de 3,7 , 14, 21 y 28 días y por cada combinación, procediéndose finalmente al curado de las probetas de concreto para su posterior ensayo de resistencia a la compresión de muestras cilíndricas de concreto.

\section{RESULTADOS}

Respecto a la calidad de los agregados gruesos de las distintas canteras se muestran los resultados de los ensayos de laboratorio se detalla a continuación en la tabla 1 .

Se estiman los resultados de los ensayos de laboratorio en la tabla 1, relacionado a la calidad del agregado grueso de la Cantera Río Mantaro, anexo Santa Rosa - Huamancaca Chico

Se estiman los resultados de los ensayos de laboratorio en la tabla 2 se aprecia los resultados de los ensayos de laboratorio respecto de la calidad de los agregados finos de canteras Río Mantaro - Puente Las Palmas.

Se estiman los resultados en la tabla 3 , de los pesos por tanda por bolsa de cemento y por metro cúbico para un diseño de mezcla teórico para fabricar un concreto de 350 $\mathrm{kg} / \mathrm{cm} 2$ de resistencia (muestra patrón).

Tabla 1. Agregado grueso: cantera Río Mantaro, Anexo Santa Rosa, Distrito Huamancaca Chico

\begin{tabular}{clccc}
\hline Ítem & Ensayos de laboratorio & Límites permisibles norma NTP 400.037 & Resultado & Observación \\
\hline 1 & Abrasión de los Ángeles & Máx. $50 \%$ & $20.32 \%$ & Cumple \\
2 & Porcentaje de una cara fracturada & No Precisa & $82.20 \%$ & No aplica \\
3 & Porcentaje de dos a más caras fracturadas & No Precisa & $88.40 \%$ & No aplica \\
4 & Inalterabilidad del agregado fino por sulfato de magnesio & Máx. $18 \%$ & $2.95 \%$ & Cumple \\
5 & Porcentaje de partículas chatas $3 / 4^{\prime \prime}$ & No Precisa & $31.12 \%$ & No aplica \\
6 & Porcentaje de particular alargadas $3 / 4^{\prime \prime}$ & No Precisa & $6.94 \%$ & No aplica \\
7 & Porcentaje de partículas chatas $1 / 2 "$ & No Precisa & $24.90 \%$ & No aplica \\
8 & Porcentaje de partículas chatas $1 / 2 "$ & No Precisa & $31.30 \%$ & No aplica \\
9 & Sulfatos en agregados & No Precisa & 548.13 ppm & No Aplica \\
\hline
\end{tabular}

Tabla 2. Agregado fino: cantera Río Mantaro, Puente Las Palmas

\begin{tabular}{|c|c|c|c|c|}
\hline Ítem & Ensayos de laboratorio & Límites permisibles norma NTP 400.037 & Resultado & Observación \\
\hline 1 & Inalterabilidad del agregado fino por sulfato de magnesio & Máx. $15 \%$ & $9.376 \%$ & Cumple \\
\hline 2 & Sales solubles en agregados & No Precisa & 720 ppm & No aplica \\
\hline 3 & Equivalente de arena & Min. $75 \%$ & $82 \%$ & Cumple \\
\hline 4 & Impurezas Orgánicas & Máx. 3 & 1 & Cumple \\
\hline 5 & Pasante por la malla Nro 200 & Máx. 5\% & $0.54 \%$ & Cumple \\
\hline 6 & Sulfatos en Agregados & No Precisa & 563.89 ppm & No aplica \\
\hline 7 & Arcilla en terrones y partículas desmenuzables (friables) en agregados & Máx. $3 \%$ & $4.00 \%$ & No cumple \\
\hline
\end{tabular}


En la tabla 4 se aprecia los resultados del diseño de mezcla práctico para fabricar un concreto de $350 \mathrm{~kg} / \mathrm{cm} 2$ de resistencia (muestra patrón), obteniéndose resultados de $495 \mathrm{~kg} / \mathrm{cm}^{2}$ de resistencia a los 28 días.

A los 28 días se obtuvieron los siguientes resultados de los diseños de mezcla de $350 \mathrm{~kg} / \mathrm{cm}^{2}$ con los reemplazos de $30 \%$ del agregado grueso y $20 \%$ del agregado fino. En las figuras siguientes se muestran los resultados de las diferentes resistencias de concreto en relación con la edad de las mismas y que obedecen a diferentes porcentajes en las cuales se han considerado diferentes composiciones de los residuos sólidos reciclados.

En la figura 1 se probó la fabricación de concreto con poliestireno extruido recuperado lavado (30\% de Agregado grueso por XPS y $20 \%$ de Agregado fino por XPS), a los 28 días de sometido al ensayo de resistencia a la compresión del concreto alcanza los $365 \mathrm{~kg} / \mathrm{cm}^{2}$. En la figura 2 se probó la fabricación de concreto con poliestireno extruido recuperado sin lavar (30\% de Agregado grueso por XPS y $20 \%$ de Agregado fino por XPS), a los 28 días la resistencia a la compresión del concreto alcanza los 330 $\mathrm{kg} / \mathrm{cm}^{2}$.

En la figura 3 se probó la fabricación de concreto con poliestireno expandido recuperado (30\% de Agregado grueso por EPS y $20 \%$ de Agregado fino por EPS). Se aprecia como a los 28 días la resistencia del concreto alcanza los $315 \mathrm{~kg} / \mathrm{cm}^{2}$. En la figura 4 y figura 5 , los resultados corresponden a la resistencia del concreto normal sin poliestireno extruido ni poliestireno expandido. Se puede apreciar que a los 28 días la resistencia del concreto alcanza $\operatorname{los} 495 \mathrm{~kg} / \mathrm{cm}^{2}$.

Los resultados de resistencia a la compresión de concreto, arriba descritos, fueron sometidos a un análisis estadístico descriptivo, cuyos resultados se muestran en la tabla 5.

En la tabla 5, se puede apreciar las medias y desviación estándar de las mezclas de diferentes porcentajes de 30\% AG y $20 \%$ AF de $30 \%$ AG y $40 \%$ AF y de $60 \%$ Ag y $70 \%$ AF

Tabla 3. Cantidad de materiales para concreto $F^{\prime} \mathrm{c}=350 \mathrm{~kg} / \mathrm{cm}^{2}$

\begin{tabular}{lc}
\hline Pesos por tanda por bolsa de cemento & \\
\hline Cemento & $42.50 \mathrm{~kg} / \mathrm{bolsa}$ \\
Agua & $15.39 \mathrm{~L} / \mathrm{bolsa}$ \\
Agregado fino húmedo (arena gruesa) & $58.49 \mathrm{~kg} / \mathrm{bolsa}$ \\
Agregado grueso húmedo (piedra chancada) & $78.51 \mathrm{~kg} / \mathrm{bolsa}$ \\
\hline Pesos por tanda por metro cúbico & \\
\hline Cemento & $506.33 \mathrm{~kg} / \mathrm{m}^{3}$ \\
Agua & $183.30 \mathrm{~L} / \mathrm{m}^{3}$ \\
Agregado fino húmedo (arena gruesa) & $696.78 \mathrm{~kg} / \mathrm{m}^{3}$ \\
Agregado grueso húmedo (piedra chancada) & $935.36 \mathrm{~kg} / \mathrm{m}^{3}$ \\
\hline
\end{tabular}

Tabla 4. Muestra Patrón $\mathrm{f}^{\prime} \mathrm{c}=350 \mathrm{~kg} / \mathrm{cm}^{2}$ Resistencia de diseño

\begin{tabular}{lcc}
\hline Especificación & Edad, días & f'c promedio $\left(\mathbf{k g} / \mathbf{c m}^{2}\right)$ \\
\hline Cantera Pilcomayo / Cantera Río Mantaro & 28 & 495 \\
\hline
\end{tabular}

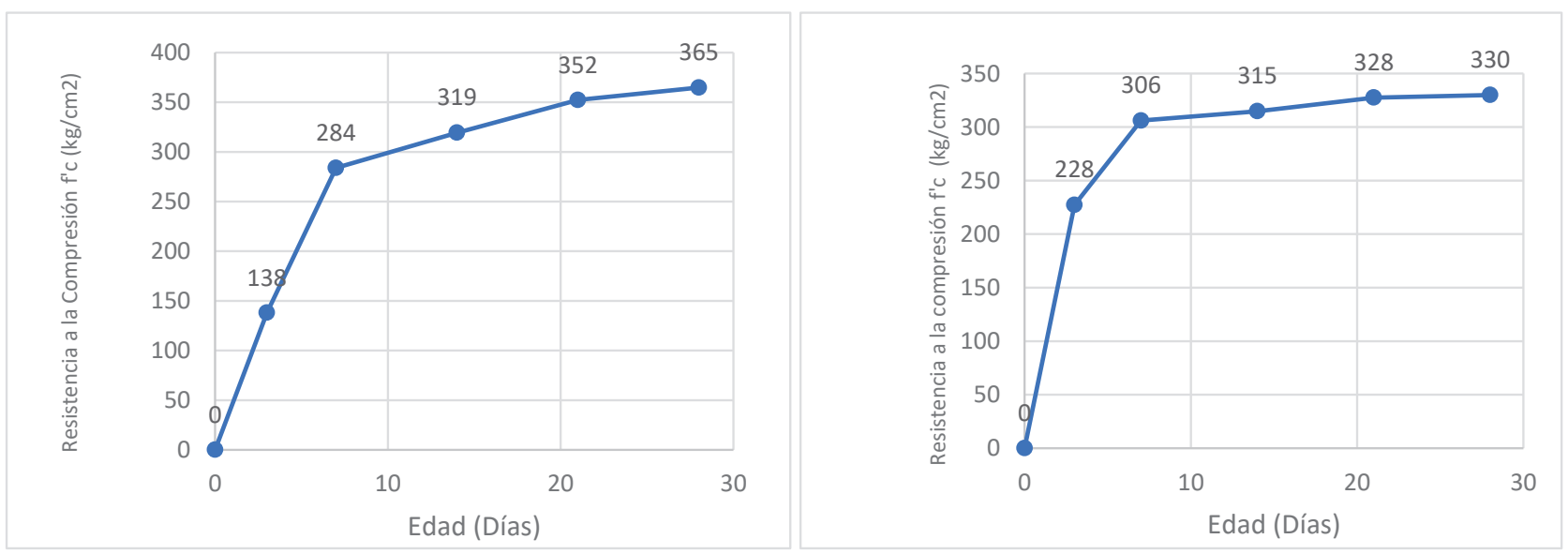

Figura 1 y Figura 2. Se aprecia la resistencia a la compresión del concreto en función de la edad. 

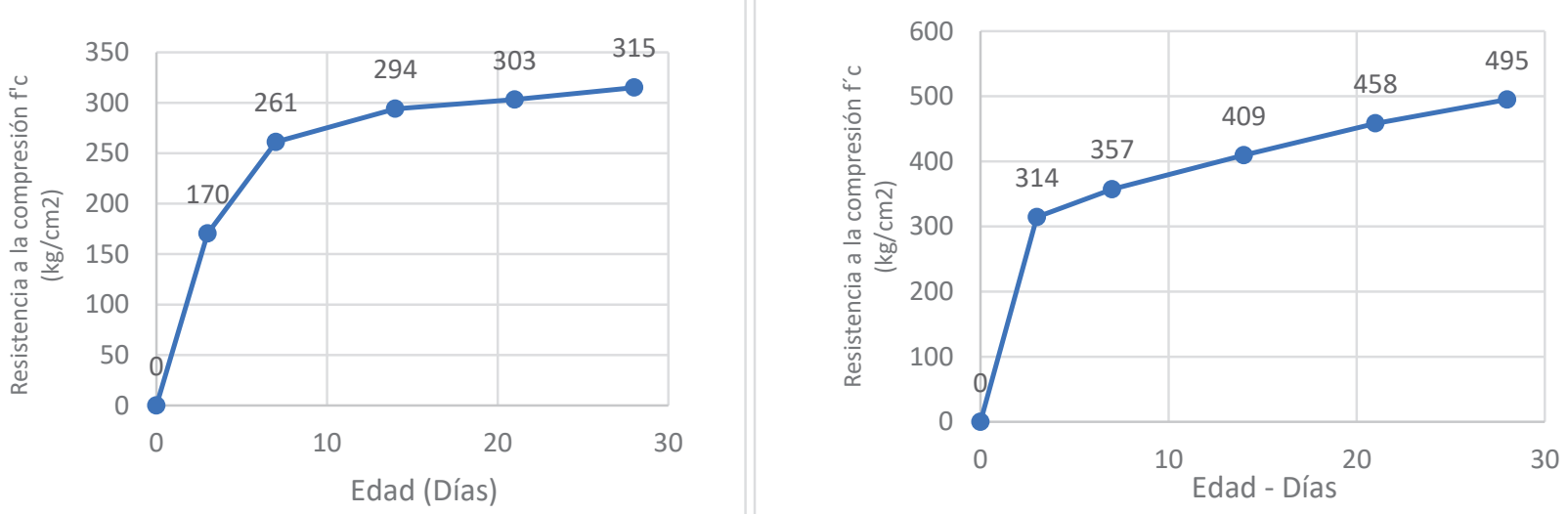

Figura 3 y Figura 4. Muestra el ensayo de resistencia a la compresión del concreto en función a la edad.
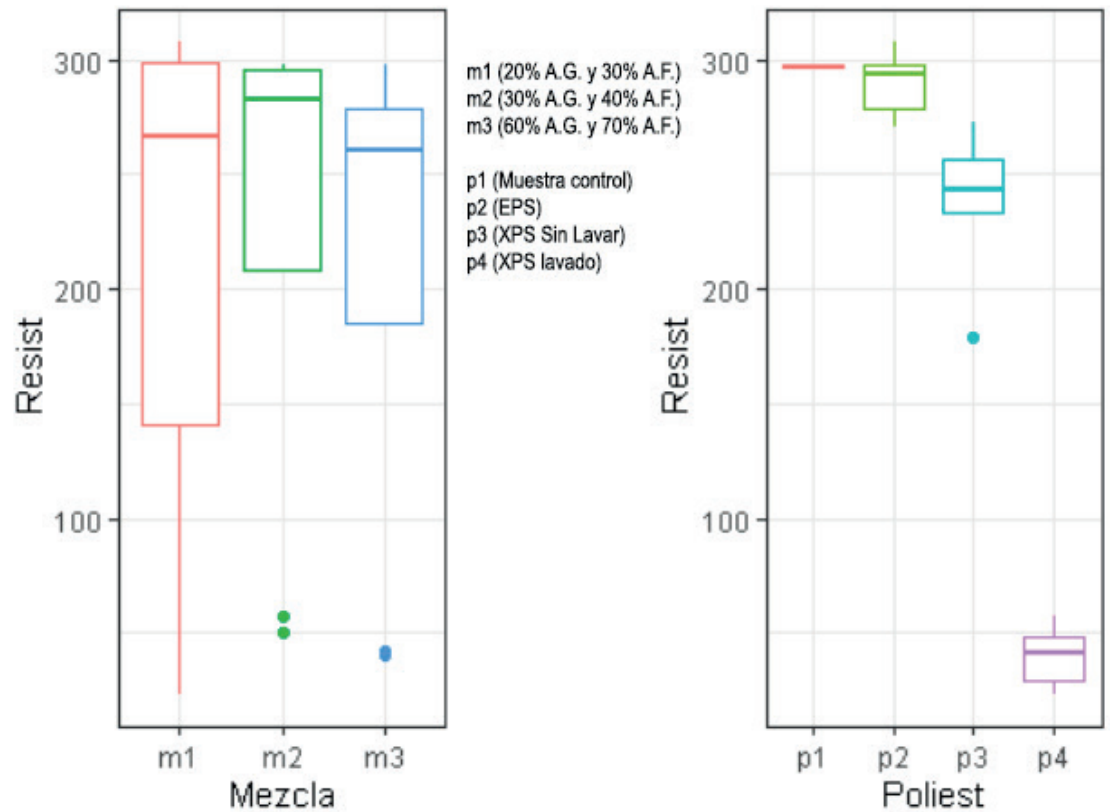

Figura 5. Resistencia a la compresión de concreto según grado de la unión de agregados (lado izquierdo) y tipo de poliestireno (lado derecho), se aprecian que la media de la resistencia de EPS (p2) supera a la media del control ( $\mathrm{p} 1$ ) en la mezcla $30 \% \mathrm{AG}$ y $20 \% \mathrm{AF}$ (m1), la media de EPS (p2) es aproximadamente igual a la media del control ( $\mathrm{p} 1$ ) en la mezcla $30 \%$ AG y $40 \%$ AF (m2); las medias de las otras combinaciones son inferiores a la media del control en las tres mezclas de agregados, tal como se pueden observar en las figuras 6 y 7 .

Tabla 5. Media y desviación estándar de la resistencia a la compresión de concreto

\begin{tabular}{llccc}
\hline Mezcla & Poliestireno & Mediciones & Media & Desviación estándar \\
\hline \multirow{3}{*}{$30 \%$ AG y 20\% AF } & Control & 2 & 297,000 & 1,4142 \\
& EPS & 2 & 302,850 & 6,7175 \\
& XPS sin lavar, SL & 2 & 293,650 & 2,3688 \\
& XPS lavado, LA & 2 & 271,800 & 17,8191 \\
30\% AG y 40\% AF & Control & 2 & 297,000 & 1,4142 \\
& EPS & 2 & 207,450 & 63,3214 \\
& XPS sin lavar, SL & 2 & 265,650 & 22,1678 \\
& XPS lavado, LA & 2 & 241,100 & 39,5273 \\
& Control & 2 & 297,000 & 1,4142 \\
& EPS & 2 & 24,300 & 192,8280 \\
& XPS sin lavar, SL & 2 & 53.750 & 172,0037 \\
& XPS lavado, LA & 2 & 41,550 & 180,6304 \\
\hline
\end{tabular}




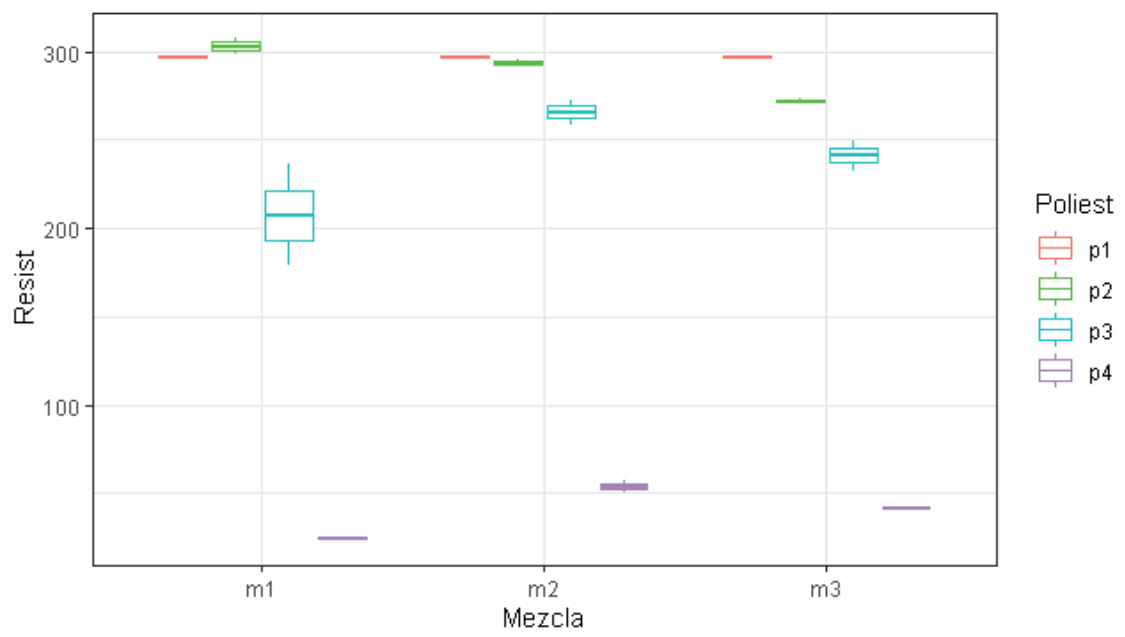

Figura 6. Resistencia a la compresión de concreto según tipo de poliestireno y grado de mezcla de agregados

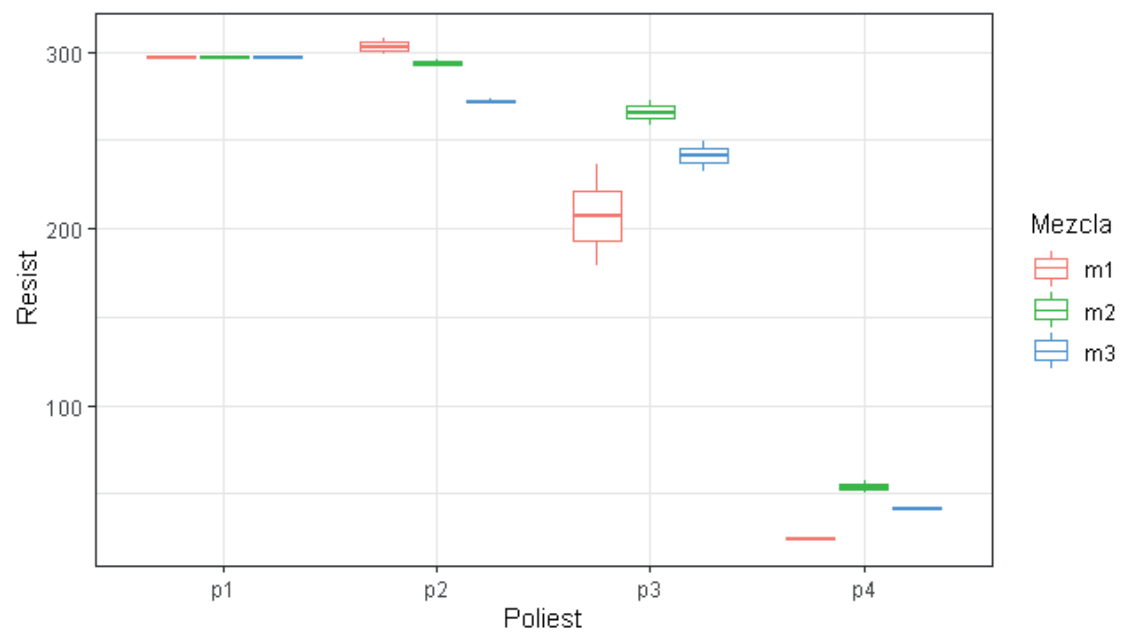

Figura 7. Resistencia a la compresión de concreto según grado de mezcla de agregados y tipo de poliestireno

con diferentes adiciones de EPS, XPS sin Lavar, XPS lavado y la muestra control.

De acuerdo con los gráficos de cajas (boxplots), se pueden apreciar que la resistencia a la compresión de concreto presenta puntos discordantes o atípicos inferiores en la mezcla $30 \%$ AG y $40 \%$ AF (m2: 2), mezcla $60 \%$ AG y $70 \%$ AF (m3: 1), y XPS SL (p3:1)

El porcentaje de contracción del EPS recuperado en la mezcla de concreto es $3 \%$ y para el XPS recuperado lavado y limpio es de $1.2 \%$

\section{DISCUSIÓN}

A continuación, se discuten los resultados estadísticos aplicados a la compresión de concretos estudiados.

\subsection{Independencia de las observaciones}

La prueba de aleatoriedad o de rachas de Wald-Wolfowitz revela que la resistencia a la compresión de concreto es una sucesión o serie no aleatoria (observaciones sistemáticas o no aleatorias), al informar el valor $\mathrm{p}$ de 0.0369 , inferior que el nivel de valor de 0.05 .

\subsection{Normalidad de las observaciones}

Dado que hay dos factores: grado de mezcla de agregados con tres niveles y tipo de poliestireno con cuatro niveles y diseño factorial balanceado $3 \times 4 \times 2(n=24$ observaciones, $\mathrm{n}<50$ ), la estadística para evaluar la normalidad de las observaciones es Shapiro-Wilk. Los resultados son de la Normalidad de la resistencia según grado de mezcla y valores p reportados por el estadístico de Shapiro-Wilk, la resistencia a la compresión de concreto no es normal, pues todos: $\mathrm{m} 1=0.0153, \mathrm{~m} 2=0.0009$ y m $3=0.0051$ son menores que el nivel de significación de 0.05. De acuerdo con la Normalidad de la resistencia según tipo de poliestireno el estadístico de Shapiro-Wilk indica sobre la resistencia a la compresión de concreto es normal para EPS (p2), XPS SL (p3) y XPS LA (p4), pero no para el control $(\mathrm{p} 1)$, al dar valores $\mathrm{p} 1=0.4208, \mathrm{p} 2=0.354$ y $\mathrm{p} 3$ $=0.5823$, mayores que el nivel de significación de 0.05 en 
EPS, XPS SL y XPS LA, y p $=0.004$ menor que este nivel en el control.

En vista que la resistencia no es normal en todos los grupos, se aplicó el criterio de homocedasticidad de las observaciones (varianzas constantes entre los grupos) a través de los estadísticos de Levene y Fligner - Killeen.

De acuerdo con la prueba de Fligner-Killeen, las varianzas a la resistencia a la compresión de concreto son homogéneas o constantes según el grado de mezcla, pero no según el tipo de poliestireno, al reportar valores $\mathrm{p}$ de 0.1566 y 0.012 , mayor y menor que el nivel de valor de 0.05 . En cambio, la evaluación de Levene indica a las varianzas de la resistencia a la compresión de concreto son homogéneas tanto según el grado de mezcla como según el tipo de poliestireno, al reportar valores $\mathrm{p}$ de 0.8612 y 0.0635 , mayores que el nivel de significación de 0.05 .

\subsection{Análisis de la influencia del poliestireno recuperado en la fabricación del concreto}

Para este propósito se realizó un análisis general (modelo lineal general univariado, ANOVA bifactorial), la resistencia a la compresión de concreto con los factores mezcla (tres niveles: $30 \%$ AG y $20 \%$ AF, $30 \%$ AG y 40 $\% \mathrm{AF}, 60 \% \mathrm{AG}$ y $70 \% \mathrm{AF}$ ) y tipo de poliestireno (cuatro niveles: control, EPS, XPS SL, XPS LA), reportado por el programa estadístico RStudio v. 1.2.5042, indica que: Existen diferencias significativas en la resistencia media según el grado de mezcla de agregados, al reportar el valor $p$ de 0,0245 , el cual es menor que el nivel de significación de 0,05 .

Se observan diferencias súper significativas en la resistencia media según el tipo de poliestireno, al mostrar la estimación $\mathrm{p}$ de 0 , inferior que el nivel de significación de 0,001 .

Se aprecian diferencias significativas en la resistencia media según la interacción grado de mezcla de agregados y tipo de poliestireno, al revelar la estimación p de 0.0246 , inferior que el nivel de significación de 0,05 .

De estos resultados se deriva que hay diferencias significativas en la media de la resistencia a la compresión de concreto, según grado de mezcla de agregados, tipo de poliestireno, y la interacción grado de mezcla de agregados y tipo de poliestireno. El tamaño del efecto de ANOVA, dada por $\eta^{2}$ es de 0.0062 para el grado de mezcla de agregados, 0.9729 para el tipo de poliestireno y 0.0136 para la interacción grado de mezcla de agregados y tipo de poliestireno, con $\eta^{2}$ parciales de $0.461,0.9926$ y 0.652 .

En las tres mezclas (m1, m2 y m3), la media de la resistencia con el poliestireno control (p1) es constante, la media de la resistencia con EPS (p2) es mayor a la media de la resistencia con XPS SL (p3) y XPS LA (p4) (González Madariaga, 2005).

Se realizaron también el análisis estadístico de comparaciones múltiples para determinar la resistencia a la compresión de concreto según grado de mezcla de agregados. El ensayo de comparaciones múltiples de Tukey indica que, al 95\% de confianza estadística, hay discrepancia elocuente entre las medias de la resistencia a la compresión de concreto de las mezclas $30 \%$ AG y $40 \% \mathrm{AF}(\mathrm{m} 2)$ y $30 \% \mathrm{AG}$ y $20 \% \mathrm{AF}(\mathrm{m} 1)$, con medias de 227.51 y $207.9 \mathrm{~kg} / \mathrm{cm}^{2}$, respectivamente, favorable a la primera mezcla, al detallar el valor $\mathrm{p}$ de 0.024 , menor que el nivel de significación de 0.05 . Además, la prueba detalla que no existen desigualdades significativas entre las medias de las mezclas $60 \% \mathrm{AG}$ y $70 \% \mathrm{AF}(\mathrm{m} 3)$ y $30 \%$ AG y $20 \%$ AF (m1) con la media de $30 \%$ AG y $40 \%$ AF (m2), al dar valores p: 0.722 y 0.092 , mayores que el nivel de significación de 0.05 .

Por otro lado, el análisis de resistencia a la compresión de concreto según tipo de poliestireno, dado que el tipo de poliestireno cuenta con un nivel control o testigo, la prueba de comparaciones

indica que hay diferencias significativas entre las medias de la resistencia a la compresión de concreto entre las interacciones $\mathrm{m} 1 \mathrm{p} 3, \mathrm{~m} 1 \mathrm{p} 4, \mathrm{~m} 2 \mathrm{p} 4, \mathrm{~m} 3 \mathrm{p} 3$ y $\mathrm{m} 3 \mathrm{p} 4$ con el control, ya que sus valores $\mathrm{p}:<0.001$ y 0.015 , son menores que el nivel de significación de 0,05 , con medias de 207.45, $24.3,53.75,241.1,41.55$ y $297 \mathrm{~kg} / \mathrm{cm}^{2}$, respectivamente, favorable al control. Las medias de la resistencia de las interacciones $\mathrm{m} 1 \mathrm{p} 2, \mathrm{~m} 2 \mathrm{p} 2, \mathrm{~m} 2 \mathrm{p} 3$ y $\mathrm{m} 3 \mathrm{p} 2,302.85$, $293.65,265.65$ y $271.8 \mathrm{~kg} / \mathrm{cm}^{2}$, respectivamente, son estadísticamente iguales a la media del control, al dar valores p $(0.999,1,0.23$ y 0.42$)$ mayores que el nivel de significación de 0.05 .

También se analizó la resistencia a la compresión del concreto según la interacción grado de mezcla de agregados y tipo de poliestireno. Para este propósito, los grados de mezcla de agregados son: $30 \% \mathrm{AG}$ y $20 \% \mathrm{AF}$ (m1), 30\% AG y $40 \% \mathrm{AF}(\mathrm{m} 2)$, y $60 \% \mathrm{AG}$ y $70 \% \mathrm{AF}(\mathrm{m} 3)$; y los tipos de poliestireno: control (cont), EPS (p2), XPS SL (p3) y XPS LA (p4). Las interacciones de estos factores son: $\mathrm{m} 1 \mathrm{p} 2, \mathrm{~m} 1 \mathrm{p} 3, \mathrm{~m} 1 \mathrm{p} 4, \mathrm{~m} 2 \mathrm{p} 2, \mathrm{~m} 2 \mathrm{p} 3, \mathrm{~m} 2 \mathrm{p} 4, \mathrm{~m} 3 \mathrm{p} 2, \mathrm{~m} 3 \mathrm{p} 3$ y m3p4. Es decir, según la prueba de Tukey, la mezcla de agregados $30 \% \mathrm{AG}$ y $40 \% \mathrm{AF}$, alcanza la media más alta de la resistencia a la compresión de concreto, $227.51 \mathrm{~kg} /$ $\mathrm{cm}^{2}$, sobre la primera $(30 \% \mathrm{AG}$ y $20 \% \mathrm{AF}$ y la mezcla 60 $\%$ AG y $70 \%$ AF, 207.9 y $212.86 \mathrm{~kg} / \mathrm{cm}^{2}$, respectivamente.

De acuerdo con la prueba de Dunnett, el poliestireno EPS logra la media de $289.43 \mathrm{~kg} / \mathrm{cm}^{2}$, igual a la media de control, $297 \mathrm{~kg} / \mathrm{cm}^{2}$, mientras que las medias de los poliestirenos XPS SL y XPS LA son inferiores a la media del control, 238.07 y $39.87 \mathrm{~kg} / \mathrm{cm}^{2}$, respectivamente.

En concordancia con la prueba de Dunnett, las interacciones $\mathrm{m} 1 \mathrm{p} 2, \mathrm{~m} 2 \mathrm{p} 2, \mathrm{~m} 2 \mathrm{p} 3$ y $\mathrm{m} 3 \mathrm{p} 2$ alcanzan medias de $302.85,293.65,265.65$ y $271.8 \mathrm{~kg} / \mathrm{cm}^{2}$, respectivamente, estadísticamente iguales a la media del control $297 \mathrm{~kg} / \mathrm{cm}^{2}$. Por su parte, las medias de las interacciones $\mathrm{m} 1 \mathrm{p} 3, \mathrm{~m} 1 \mathrm{p} 4$, m2p3, m3p3 y m3p4, 207.45, 24.3, 53.75, 241.1 y $41.55 \mathrm{~kg} /$ $\mathrm{cm} 2$, respectivamente, son menores a la media del control.

(Vidal Almonacid, 2010) en su tesis sostiene que, para obtener un hormigón muy liviano, se tiene que reemplazar un alto porcentaje de poliestireno expandido con arena (Vidal Almonacid, 2010); que por ello, con una dureza disminuido habiendo la guía de diseño investigado de 240 $\mathrm{kg} / \mathrm{cm} 2$, por lo tanto no cumple con la resistencia optimo 
deseado de un diseño de hormigón para losas alivianadas, en la investigación realizada con el reemplazo del agregado grueso en un $30 \%$ y agregado fino en un $20 \%$ por el poliestireno extruido lavado se supera la resistencia de $350 \mathrm{~kg} / \mathrm{cm} 2$, sin embargo con otros reemplazos no se logra superar la resistencia de diseño.

\section{CONCLUSIÓN}

El acondicionamiento preliminar del poliestireno reciclado influye en la resistencia a la compresión de concreto, se ha demostrado que la fabricación de concreto formulado con poliestireno extruido reciclado y previamente sometido a limpieza por lavado con agua, cuya mezcla es de $30 \%$ de agregado grueso por poliestireno extruido (XPS) y 20 $\%$ de agregado fino por XPS. a la edad de 28 días, supera la resistencia de diseño de $350 \mathrm{~kg} / \mathrm{cm} 2$, obteniéndose un valor de $365 \mathrm{~kg} / \mathrm{cm} 2$, valor que supera el límite permisible de la norma técnica y representa aproximadamente un 74 $\%$ respecto de la calidad de concreto sin formulación con residuos sólidos cuyo valor es $495 \mathrm{~kg} / \mathrm{cm} 2$ (Rojas, 2014). Para mayores resistencias del concreto con EPS y XPS recuperados se podría evaluar el uso de plastificantes y/o superplastificantes a fin de disminuir la cantidad de agua y aumentar la resistencia del concreto con poliestirenos recuperados.

Las otras formulaciones de concreto que incorporan combinaciones con poliestireno extruido sin previo tratamiento de limpieza por lavado y el poliestireno expandido sometido sólo a limpieza superficial que elimina material particulado, no superaron la resistencia de diseño.

\section{AGRADECIMIENTOS}

Nuestro agradecimiento a la Empresa Inversiones Generales Centauro Ingenieros S.A.C. por brindarnos las instalaciones de su laboratorio del área de concreto en la ciudad de Huancayo para los análisis relacionados a esta investigación.

\section{REFERENCIAS}

Álvarez, C. (2010). La bandeja blanca de poliestireno Ecolaboratorio. Diario El País España. Blogs El País Semanal. https://blogs.elpais.com/eco-lab/2010/10/labandeja-blanca-de-poliestireno.html

BETANCOURT S. Daylin Julieth, \& SOLANO M, J. K. (2016). SYNTHESIS AND CHARACTERIZATION OF EXPANDED MIXED POLYSTYRENE POLYPROPYLENE (STYROFOAM) RECYCLED AS AN ALTERNATIVE FOR PRODUCTION PROCESS OF AUTOPARTS. Luna Azul, 43(2), 286310 . http://www.scielo.org.co/scielo.php?pid=S1909$24742016000200013 \&$ script $=$ sci_abstract\&tlng=en
Betancourt Sánchez, D. J. (2015). Aprovechamiento del poliestireno expandido (icopor) reciclado como alternativa a la fibra de vidrio en el proceso de producción de autopartes en la empresa ventiladores $G B A$. https://repository.usta.edu. co/handle/11634/2909

Carolina Montes Cortés. (2009). Régimen jurídico y ambiental de los residuos sólidos. Universidad Externado de Colombia. Facultad de Derecho, 1(166). https://ideas.repec.org/b/ext/ derech/166.html

González Madariaga, F. J. (2005). Caracterización de mezclas de residuos de poliestireno expandido (EPS) conglomerados con yeso o escayola, su uso en la construcción. https:// upcommons.upc.edu/handle/2117/94156

McCauley, D. J. (2015). Marine defaunation: Animal loss in the global ocean. Science, 347(6219). https://www.science.org/ lookup/doi/10.1126/science.1255641

Natura. (2019). Fundación Verde Natura. http:// fundacionverdenatura.org/proceso-de-reciclaje.html

OEFA. (2014). Fiscalización ambiental en residuos sólidos de gestión municipal provincial: informe 2013-2014. Índice de cumplimiento de los municipios provinciales a nivel nacional. Organismo de Evaluación y Fiscalización Ambiental . https://repositorio.oefa.gob.pe/handle/20.500.12788/56

Parres García, F. (2005). Investigación de las variables limitantes en la recuperación de los residuos de poliestireno procedentes del sector envase. https://riunet.upv.es/ handle/10251/1852

Rodríguez, N., Avellaneda, L., \& Zerda, D. (2014). Estudio de factibilidad para la recolección, acopio, molido y comercialización de PET (Polietilen Teleftarato) en el municipio de Soacha "Repetmol S.A.S." In Corporación Universitaria Minuto de Dios. https://repository.uniminuto. edu/handle/10656/3147

Rojas, J. (2014). Residuos sólidos y calentamiento global - parte 1. Exito Empresarial, 254, 1-3. http://www.cegesti.org/ exitoempresarial/publicaciones/publicacion_254_310314 es.pdf

Vidal Almonacid, F. G. (2010). Caracterización y Evaluación del Comportamiento de Hormigones Livianos, Usando como materia prima Poliestireno Expandido Modificado (MEPS). http://cybertesis.uach.cl/tesis/uach/2010/bmfciv648c/doc/ bmfciv648c.pdf

Villafuerte, I. , Flores, D. , Guadalupe, E., \& Zea, M. (2014). Evaluación ambiental del relleno sanitario para el santuario histórico de Machu Picchu y pueblos aledaños. Revista Del Instituto de Investigación de La Facultad de Minas, Metalurgia y Ciencias Geográficas, 7(14). https:// revistasinvestigacion.unmsm.edu.pe/index.php/iigeo/ article/view/732 